\section{La lectura de artículos científicos. Entrevista a estudiantes del pregrado}

\section{The reading of scientific articles. Interview to undergraduate students}

\section{Entrevista}

\author{
Yuri Castro-Rodríguez ${ }^{1, a}$ \\ 1 Universidad Nacional Mayor de San Marcos, Especialidad \\ de Periodoncia e Implantología, Lima, Perú. \\ a Magíster en Educación.

\section{Correspondencia:} \\ Yuri Castro Rodríguez: yuricastro_16@hotmail.com \\ Jr. Tomás Catari 463. Urb. El trébol. Los Olivos. Lima, Perú. \\ ORCID: 0000-0002-9587-520X
}

Conflicto de intereses: el autor declara no tener conflictos de interés.

\section{Fuente de financiamiento: autofinanciado.}

Recibido: 23/06/19

Aceptado: 28/10/19

Publicado: 24/02/20

\begin{abstract}
Resumen
La lectura de artículos científicos es parte del hábito de un investigador y en el caso de los estudiantes es una estrategia de enseñanza-aprendizaje para que se familiarice con el conocimiento científico difundido, estilos de redacción, manejo de casos clínicos y comprensión de métodos. En la presente entrevista se recopilan las opiniones de estudiantes sudamericanos de tres facultades de Odontología (Universidad Nacional Mayor de San Marcos-Perú, Universidad de San Sebastián-Chile y Universidad Nacional de Córdoba-Argentina) respecto a la lectura y uso de las revistas científicas. La información recabada permite explorar las percepciones que tienen los estudiantes sobre la lectura de artículos científicos.
\end{abstract}

Palabras clave: Investigación; Entrevista; Lectura; Estudiantes de Odontología (fuente: DeCS BIREME).

\begin{abstract}
Scientific articles reading is part of a researcher habit. Meanwhile, for students it is a teaching-learning strategy to familiarize themselves with the widespread scientific knowledge, writing styles, clinical case management and methods comprehension. In the present interview the opinion of South American students from three dental faculties (Universidad Nacional de San Marcos-Perú, Universidad de San Sebastián-Chile and Universidad Nacional de Córdoba-Argentina) are compiled regarding the reading and usefulness of scientific journals. The information collected allows us to explore the student perceptions about scientific articles reading.
\end{abstract}

Keywords: Research; Interview; Reading; Students, dental (source: MeSH NLM). 


\section{Introducción}

En las ciencias de la salud, la enseńanza de la investigación favorece las decisiones clínicas que tome el futuro profesional. De esta forma, el primer acercamiento a un paciente permite poner a prueba las técnicas comunicativas (la entrevista como técnica de recolección de datos 1), la exploración de signos y síntomas permite recolectar datos (implica hacer uso de la observación e índices clinimétricos ${ }^{2}$ ), la búsqueda de un diagnóstico requiere de un pensamiento crítico (análisis de un problema y búsqueda de la solución), la selección e indicación de exámenes auxiliares permite utilizar técnicas de recolección de datos, el planteamiento de un tratamiento y el seguimiento clínico permiten analizar resultados e interpretar datos en el posoperatorio, y finalmente sintetizar los hallazgos en una epicrisis permite redactar un informe final y comunicarlo a través de un medio formal ${ }^{3}$. La investigación científica y la práctica clínica estudiantil van de la mano puesto que son parte de las actividades a realizar durante el examen clínico, el diagnóstico, el tratamiento y el seguimiento del paciente.

De aquí la importancia de la enseñanza de la investigación científica en el pregrado. Parte de esta enseñanza y la adquisición de competencias investigativas se logra a partir de la lectura y análisis de artículos científicos publicados en revistas. A partir de la lectura de los mismos, los estudiantes acceden a información científica actualizada, comprenden los estilos de redacción, analizan casos clínicos similares a los que atienden en su práctica pre profesional, sienten curiosidad por publicar artículos científicos y conocen los procesos de producción y edición de manuscritos.

En la Odontología este modelo de enseñanza en base a artículos científicos ha venido creciendo tanto a nivel de posgrado como de pregrado. La conocida "Odontología basada en evidencia" ha permitido introducir el análisis de artículos científicos en los planes de estudio. Asimismo, resulta interesante comprender cómo estas estrategias de enseñanza se han venido utilizando en distintos espacios de aprendizaje a nivel latinoamericano, también resulta llamativo conocer la perspectiva del estudiante respecto al uso de los artículos científicos dentro de las actividades de enseñanza-aprendizaje y evaluación. En la búsqueda de esta información se ha entrevistado a tres estudiantes del pregrado de Odontología/Estomatología de tres países sudamericanos (Argentina, Chile y Perú): Julian Doba, estudiante de 3er año de la Universidad Nacional de Córdoba-Argentina (la carrera de Odontología demanda estudios por cinco años); Leonardo Meza Palma, estudiante de 5to año de la Universidad de San Sebastián-Chile (universidad privada donde la carrera de Odontología demanda estudios por cinco años); y Claudia Piscoche Rodríguez, estudiante de 5to año de la Universidad Nacional Mayor de San Marcos-Perú (la carrera de Odontología demanda estudios por seis años). Los estudiantes fueron seleccionados por conveniencia quienes respondieron favorablemente a un llamado por correo electrónico para aceptar ser parte de la entrevista, la cual se realizó vía online a través de una guía semiestructurada entre los meses de mayo a junio del 2019.

El objetivo de la entrevista fue indagar las perspectivas que tienen los estudiantes sobre el uso de artículos y revistas científicas durante sus actividades del pregrado.

\section{Entrevista}

¿Durante las clases del pregrado, qué exigencias presentaste en cuanto a lectura de artículos científicos?

Julian Doba (JD): En nuestra facultad, hay ciertas materias en la cual nos han pedido la lectura de ciertos papers o artículos académicos, no así resumirlos o realizar gráficos o estadísticas. En primer año del pregrado, una de las asignaturas fue Metodología de la investigación, donde pudimos leer varios artículos y discutir sobre ellos.

Leonardo Meza Palma (LMP): Todas las clases se basaban en papers, dependía del curso la modalidad de trabajo, en algunos solamente era leerlos, otros se realizaba trabajo grupal contestando preguntas enfocadas en el artículo, a veces teníamos que realizar resúmenes y una exposición oral de lo más importante del paper. El área que más exigió esta modalidad fue Periodoncia II (5to año).

Claudia Piscoche Rodríguez (CPR): Para el desarrollo de mis asignaturas sí se exige leer artículos científicos, por ejemplo, en uno de mis cursos que es clínico nos piden exponer sobre el caso del paciente a tratar y al último colocar cuatro artículos relacionados al caso del paciente, explicándole mediante un mapa conceptual, de esta manera uno como estudiante tiene la necesidad de leer detalladamente sobre el tema. Uno de los cursos más exigentes en el análisis de artículos es el de Odontopediatría.

\section{¿Dónde suelen buscar los artículos cientificos?}

JD: El buscador que más utilizo diariamente es SciELO. En algunos casos puedo descargarlos y otros tantos, no. Es de acceso gratuito sino podemos recurrir a un docente para que nos descargue el mismo. Generalmente los buscamos en idioma inglés o español. En la mayoría de veces leemos todo el artículo y en otras solo la parte en la que estoy enfocado.

LMP: En base de datos, la universidad tiene una plataforma donde tenemos libre acceso a muchas bases de datos para descarga de material, en el caso que el paper no esté gratuito, se puede solicitar a la universidad que haga el contacto con el autor del paper y lo consiga. Por lo general los artículos son en inglés, español, pero si hay alguno de otro idioma se traduce el documento. Siempre leo primero el resumen para ver si va acorde a la búsqueda y de ser así leerlo completo.

CPR: Personalmente uso bases de datos como EBSCO, SciELO y Pubmed. Busco artículos de los journals, muchos de ellos no se pueden descargar y se tiene que acceder solo si se hace un pago por derecho de autor. En caso no pueda acceder a todo el artículo, leo su resumen, solo en ese caso. La mayoría de los artículos los encuentro en inglés o portugués. 

¿Los artículos que han podido leer, les fueron útiles para su
quehacer académico?

JD: En su mayoría son comprensibles, pero exageran con demasiados datos estadísticos; sin embargo, considero que esos datos siempre son de utilidad para el quehacer académico.

LMP: Sí, uno que otro paper era más denso, pero conversando con los compañeros salíamos de las dudas. En todos los papers siempre hay algo que se puede utilizar para la práctica clínica. Ciertamente el compañero que leía sólo el paper recomendado no lo hacía tener una visión crítica respecto al tema, pocos leían más papers para tener una opinión un poco más extendida.

CPR: Muchas veces en mi búsqueda por conocer más sobre un tema en específico los primeros artículos que leo no son muy bien comprendidos, pero después de unos 3 o 4 el tema y los términos se me hacen más familiares, y vuelvo a leer los primeros para una mejor comprensión. Para mi práctica clínica sí me ayudó bastante leer los artículos científicos, sobre todo los reportes de casos, porque se evidencia con fotos el tratamiento de un caso, realizan protocolos, los cuales puedes discutir comparando con otros estudios.

¿Cómo se desarrollan (difunden) las revistas cientificas de tu facultad/universidad?

JD: No conozco revistas de mi facultad. Sí tengo entendido que varios de mis docentes publican artículos y tesis que nos fueron útiles para trabajos o presentaciones en congresos estudiantiles.

LMP: La Universidad San Sebastián no tiene revista científica. Tiene Sociedad Científica de Estudiantes donde al final terminas leyendo los papers que presentan los expositores en los congresos o estudiantes en la competencia de posters.

CPR: Sí conozco la revista de mi facultad, no suelo leerlo, pero las pocas veces que pude hacerlo encontré secciones interesantes.
¿Qué revistas externas a tu universidadlfacultad has podido
conocer/leer?

JD: Hasta el momento no he podido leer ninguna revista externa a la facultad.

LMP: Muchas, no tengo una revista favorita, la que podría mencionar es la Revista Clínica de Periodoncia, Implantología y Rehabilitación Oral (PIRO) por el área de interés que tengo. Creo que es de fácil lectura. Lo aprendido principalmente son tips y/o protocolos clínicos nuevos.

CPR: Revistas extranjeras como por ejemplo las de México donde pude encontrar dos artículos muy interesantes relacionados a mi tema de tesis. En otras revistas que también pude revisar artículos no tuve dificultad para adquirir el PDF, la descarga era gratuita.

Las opiniones recabadas por los estudiantes nos permiten obtener algunas perspectivas iniciales como el hecho que utilicen el término "paper" para referirse a un artículo científico, considerar el uso de las bases de datos de sus facultades/universidades para acceder a ellos, leerlos principalmente en idioma español e inglés, enfocarse en la totalidad del manuscrito, así como considerar que son útiles para su quehacer académico.

Consideramos fundamental que se continúe con las estrategias de incluir el análisis de artículos y revistas científicas dentro del proceso enseñanza-aprendizaje de los estudiantes puesto que les permitirá generar un mayor criterio científico al momento de tomar decisiones clínicas.

\section{Referencias bibliográficas}

1. Troncoso-Pantoja C, Amaya-Placencia A. Entrevista: guía práctica para la recolección de datos cualitativos en investigación de salud. Rev Fac Med. 2017;65(2):329-32.

2. Lara-Muñoz C, Ortega-Soto H. ¿La clinimetría o la psicometría? Medición en la práctica psiquiátrica. Salud Mental. 1995;18(4):33-40.

3. Kuschel RC, Konrad SD, Cárcamo IC. Análisis crítico del registro de epicrisis del Equipo de Cirugía Digestiva Hospital Base Valdivia. Cuad Cir. 2007;21:22-30. 
